\title{
On nonclassical analytical solutions for advective transport problems
}

\author{
A. P. S. Selvadurai ${ }^{1}$ \\ Received 1 July 2007; revised 23 December 2007; accepted 5 March 2008; published 19 July 2008.
}

[1] The paper presents certain nonclassical analytical solutions for describing the onedimensional advective transport of a chemical or a contaminant in a fluid-saturated porous medium. The advective transport is induced by potential flow. The nonclassical nature stems from the influences of time-dependent boundary concentrations, time-dependent flow velocities, and the presence of natural attenuation. These analytical results are novel and provide a useful set of solutions for benchmarking computational schemes that have been developed in the literature for solving transport of chemicals and contaminants in fluid-saturated porous media.

Citation: Selvadurai, A. P. S. (2008), On nonclassical analytical solutions for advective transport problems, Water Resour. Res., 44, W00C03, doi:10.1029/2007WR006312.

\section{Introduction}

[2] The transport of chemicals, contaminants and particulates in the geosphere due to groundwater movement is an important component in the assessment of geoenvironmental hazards associated with human activity in the form of pollutant spills, release of leachates from contaminated landfill, discharge of toxic materials from mine waste tailings ponds, agricultural pollution due to fertilizer use and aquifer contamination due to migration of bacteria and fine particles [Ogata and Banks, 1961; Lindstrom et al., 1967; Bear, 1972; Greenkorn, 1983; Rubin, 1983; McDowell-Boyer et al., 1986; de Marsily, 1986; Bear and Verruijt, 1990; Bear and Bachmat, 1992; Fetter, 1993; Appelo and Postma, 1993; Banks, 1994; Vukovich, 1997; Ingham and Pop, 1998; Domenico and Schwartz, 1998; Bedient et al., 1999; Massel, 1999; Charbeneau, 2000; Vulliet et al., 2002; Selvadurai, 2006]. In addition, natural release of chemicals and their resulting transport can result from changes in groundwater flow patterns, created by construction of hydro-reservoirs, influences of climate change and from the extraction of water and energy resources, including oil, natural gas and coal [Philips, 1991; Oelkers, 1996; Ingebritsen and Sanford, 1998]. The complete study of chemical and contaminant transport in fluid-saturated media becomes a very complex problem when aspects of chemical influences on the transport and mechanical properties are taken into consideration. In many instances, the interactions and the resulting changes cannot be defined with precision, largely owing to the wide variety of geochemical reactions that can take place at different rates, an absence of information on the migrating chemical species, the geochemistry of the porous medium and the attenuation characteristics of the

\footnotetext{
${ }^{1}$ Department of Civil Engineering and Applied Mechanics, McGill University, West Montreal, Quebec, Canada.

Copyright 2008 by the American Geophysical Union. 0043-1397/08/2007WR006312\$09.00
}

porous medium itself. The prudent approach has been to consider canonical models of chemical and contaminant transport through porous media so that the parameters required to obtain results from the modeling are kept to a minimum. This allows more attention to be focused on the variabilities in the basic parameters that are perhaps more relevant for practical use and environmental decision making. The typical processes of advection, diffusion, attenuation, etc., are clearly such canonical representations of complex transport processes of chemicals and contaminants in the geosphere. When such basic processes concerning the transport of chemicals in a porous medium are identified for a particular geoenvironmental problem, the issue of hydromechanical movement within the domain of practical interest has to be examined by recourse to reliable computational techniques. In this regard, the purely advective transport of the species in the absence of diffusive-dispersive phenomena governed by concentration gradients or hydrodynamic effects, presents an extreme test case for assessing the reliability and accuracy of any sophisticated computational scheme. The research dealing with the computational modeling of the purely advective transport problem is extensive and no attempt will be made to provide a comprehensive survey of the field. The reader is referred to the volumes by Huyakorn and Pinder [1983], Bear and Bachmat [1992], Sun [1996] and Zheng and Bennett [2002] and the articles by $Y u$ and Heinrich [1986], Oñate [1998], Pereira and Pereira [2001], Hauke and Doweidar [2005] and Selvadurai and Dong [2006a, 2006b] for further articles dealing with the computational modeling of both advective and advective-diffusive transport problems. In addition, finite volume methods and particle tracking techniques [Neuman, 1981, 1984; Douglas and Russell, 1982; Morton, 1998; LeVeque, 2002] and boundary element methods [Taigbenu, 1999; Young et al., 2000; Singh and Tanaka, 2000; Boztosun and Charafi, 2002] have also been applied to investigate both advective and advective-diffusive phenomena.

[3] The standard analytical solution used for purposes of calibrating computational schemes is usually the advective 
transport in a one-dimensional region of finite extent, in the presence of a flow velocity that is temporally constant and a boundary concentration that remains time invariant. Such a standard analytical solution has been used by Noorishad et al. [1992] and Wang and Hutter [2001] to test the accuracy of several computational schemes proposed for analyzing the purely advective transport problem. Only recently have analytical solutions been developed to examine two-dimensional and axisymmetric situations involving advective transport problems [Selvadurai, 2002, 2003, 2004a, 2004b, 2006]. Even with these solutions, simplifications have to be made with respect to the time-dependency in the flow velocities and boundary concentrations to enable the development of the relevant analytical solutions. The rationale for focusing on the purely advective transport problem is clear; the absence of a diffusive term in the governing partial differential equation implies the presence of discontinuous chemical concentration profiles as the front moves through the porous medium. The performance of a computational scheme is judged in particular by its ability to model this discontinuous propagation front without numerical diffusion, oscillations and, most importantly, without the development of negative concentrations in the numerical solution. The standard one-dimensional solution used to calibrate numerical schemes is open to improvement, particularly through the incorporation of features that can account for time-dependent flow velocities and concentration boundary conditions that can exhibit time variability. Such extensions are considered useful additions to the catalog of benchmarking exercises, particularly since the time discretization algorithms in the numerical schemes have also to account for time variability in the dependent variable, namely the chemical concentration. Surprisingly such extensions are not available in the literature. The purpose of this paper is to present a catalog of exact closed form analytical solutions that can be used to test the accuracy of computational algorithms available in the literature for the solution of advection dominated transport phenomena in porous media.

\section{Governing Equations}

[4] We consider a cylindrical prismatic region of a fluidsaturated porous medium of arbitrary cross section where the plane ends are perpendicular to the axis of the region. The Dupuit-Forchheimer hydraulic conductivity of the porous medium is denoted by $k$. Flow in the porous medium can be initiated by imposing boundary potentials at the plane ends. When constant Dirichlet boundary conditions are prescribed for the flow potential at the plane ends and homogeneous Neumann conditions are prescribed on the prismatic surface, the flow is one-dimensional irrespective of the cross-sectional shape of the prismatic cylindrical region [Weinberger, 1965; Selvadurai, 2000]. Therefore the one-dimensional problem has applications to threedimensional geometry of a computational domain. Considering Darcy flow in the porous medium, the flow velocity $\mathbf{v}(\mathbf{x})$ is given by

$$
\mathbf{v}(\mathbf{x})=-k \nabla \varphi(\mathbf{x})
$$

where $\nabla$ is the gradient operator, $\mathbf{x}$ is a position vector and $\varphi(\mathbf{x})$ is the flow potential. Considering fluid mass conservation, we can show that

$$
\nabla^{2} \varphi(\mathbf{x})=0
$$

where $\nabla^{2}$ is Laplace's operator. For potential flow to take place in the one-dimensional porous medium, the flow domain should be finite. Note that the steady state flow velocities can exhibit a time-dependency only if the constant boundary potentials over the cross-sectional plane ends of the one-dimensional domain exhibit time variations. This type of flow can be encountered in a "falling head' type one-dimensional device for measuring the permeability of a porous medium.

[5] We now assume that a chemical introduced into the porous medium moves with the fluid without altering its mass density and transport properties. The equation of mass conservation for the chemical species gives the advective transport equation

$$
\frac{\partial C}{\partial t}+\nabla \cdot(C \mathbf{v})=-\xi C
$$

where $C(\mathbf{x}, t)$ is the chemical or contaminant concentration per unit volume of the fluid, $\xi$ is an attenuation (decay) factor and $\nabla$. ( ) is the divergence operator. The velocities in (3) are determined from (1) and the advective transport problem requires the solution of (3) subject to suitable initial and boundary conditions. The uniqueness of the solution to this linear initial boundary value problem is discussed by Selvadurai [2004c].

\section{Analytical Results}

[6] The development of analytical solutions for linear, hyperbolic initial boundary value problems is standard fare in pure and applied mathematics. The methods applicable to the development of analytical solutions range from the method of characteristics to integral transform techniques that use Laplace transform techniques extensively. While these methods are certainly relevant to the development of classical analytical results, they are not considered routine techniques for situations involving unconventional boundary conditions, particularly those that are time-dependent. In these circumstances, it is possible to consider trial function approaches, and suitable transformations that will reduce the hyperbolic problem to the solution of a single ordinary differential equation. A technique of this type was used by Selvadurai [2004b] to develop an exact closed form solution to an initial value problem associated with the advective transport equation. The approach adopted in the development of the present catalog of solutions for these nonconventional linear hyperbolic initial boundary value problems basically follows the heuristic approaches advocated by Selvadurai [2004b]. The final requirement for the solution is that it must be void of any requirement for extensive computations for the development of the analytical result; i.e., the solution should be in exact closed form. The availability of a catalog of exact closed form solutions, albeit for the linear advective transport equation, particularly 
with time-dependent boundary concentrations and timedependent velocities, presents an added dimension to calibration exercises, since the nondimensional Courant Number $(\mathrm{Cr}=|v| \Delta t / h$, where $|v|$ is the velocity, $\Delta t$ is the time step and $h$ is a characteristic length) and the Peclet Number $(P e=|v| h / D$, where $D$ is a diffusion coefficient) will now be time-dependent. Analytical solutions have always provided useful approaches not only for benchmarking computational approaches but also as a means of conducting preliminary calculations in the absence of precise data or data that can exhibit wide variability. The earlier work of Ogata and Banks [1961], Lindstrom et al. [1967], Lindstrom and Stone [1974a, 1974b], Lindstrom and Oberhettinger [1975], van Genuchten [1981] (see also Pang and Hunt [2001] for other references) develop a catalog of solutions relevant to the advection-diffusion problem but largely focus on flow velocities which are constant. The solutions developed in this article introduce a time-dependent velocity which is an extension that will be important to the calibration exercises.

[7] We now consider the one-dimensional equivalent of (3), where the spatial coordinate is assumed to be in the $x$ direction. The reduced advective transport equation is given by

$$
\frac{\partial C}{\partial t}+v(t) \frac{\partial C}{\partial x}=-\xi C
$$

The initial boundary value problem is solved by considering the governing equation (4), subject to different types of boundary conditions and different time-dependent variations in the spatially constant advective flow velocity.

\subsection{Plug Flow Problem}

[8] This is the standard result where the initial boundary value problem is defined by the partial differential equation

$$
\frac{\partial C}{\partial t}+v_{0} \frac{\partial C}{\partial x}=0
$$

subject to the boundary condition

$$
C(0, t)=C_{0} \mathrm{H}(t)
$$

where $\mathrm{H}(t)$ is the Heaviside step function, $C_{0}$ is a constant and $v_{0}$ is the advective flow velocity. For a contaminant-free initial state, the initial condition takes the form

$$
C(x, 0)=0 \text {. }
$$

As indicated, the initial boundary value problem posed by (5) to (7) can be solved in a variety of ways, including the method of characteristics applicable to hyperbolic equations as well as through the use of Laplace transform techniques [Selvadurai, 2000]. The exact closed form solution to the basic plug flow problem is given by

$$
C(x, t)=C_{0} \mathrm{H}\left(t-\frac{x}{v_{0}}\right) .
$$

\subsection{Plug Flow Problem With Natural Attenuation}

[9] In this case the partial differential equation is given by

$$
\frac{\partial C}{\partial t}+v_{0} \frac{\partial C}{\partial x}=-\xi C
$$

and the boundary and initial conditions are given by (6) and (7) respectively. The exact closed form solution obtained using the methods described previously is

$$
C(x, t)=C_{0} \exp \left(-\frac{\xi x}{v_{0}}\right) \mathrm{H}\left(t-\frac{x}{v_{0}}\right) .
$$

\subsection{Plug Flow Problem With Attenuation and Exponentially Decaying Boundary Concentration}

[10] The partial differential equation and the initial condition governing the advective transport problem are identical to (9) and (7) respectively and the boundary condition takes the form

$$
C(0, t)=C_{0} \exp (-\beta t)
$$

where $\beta$ is a constant. The exact closed form solution to the initial boundary value problem posed by (9), (7) and (11) takes the form

$$
C(x, t)=C_{0} \exp \left(-\left[t-\frac{x}{v_{0}}\right] \beta-\frac{\xi x}{v_{0}}\right) \mathrm{H}\left(t-\frac{x}{v_{0}}\right) .
$$

As $\beta \rightarrow 0$, this result reduces to (10).

\subsection{Plug Flow Problem for a Velocity Field With Exponential Time Decay}

[11] The initial boundary value problem is defined by the partial differential equation

$$
\frac{\partial C}{\partial t}+v_{0} \exp (-\lambda t) \frac{\partial C}{\partial x}=0
$$

$$
C(x, t)=C_{0} \mathrm{H}\left(\frac{\{1-\exp (-\lambda t)\}}{\lambda}-\frac{x}{v_{0}}\right) .
$$

\subsection{Plug Flow Problem for a Velocity Field With Exponential Time Decay, Natural Attenuation, and} Natural Attenuation of Boundary Concentration

[12] The partial differential equation governing the advective transport problem is given by

$$
\frac{\partial C}{\partial t}+v_{0} \exp (-\lambda t) \frac{\partial C}{\partial x}=-\xi C
$$

The initial condition is given by (7) and the boundary concentration varies with time according to

$$
C(0, t)=C_{0} \exp (-\xi t) .
$$




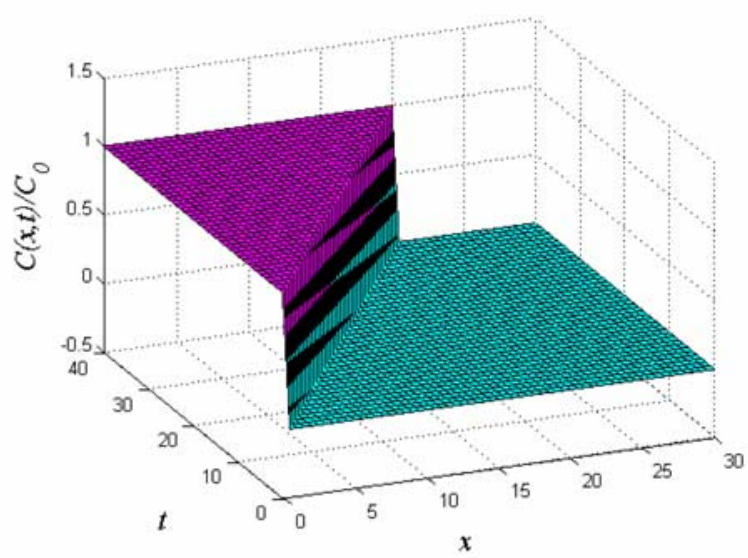

Analytical solution [Eq. (8)]

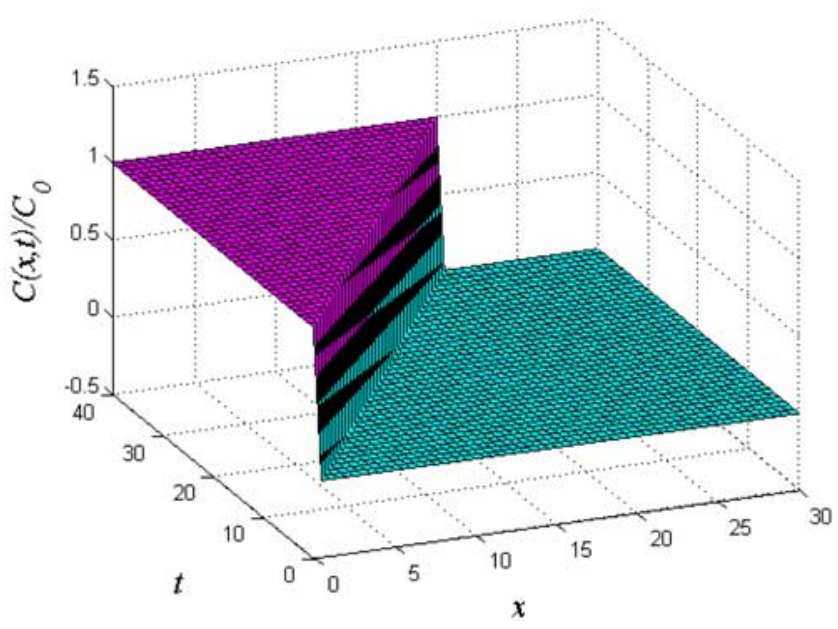

MLS with $\alpha=3 / 2$ and $\theta=1 / 3$

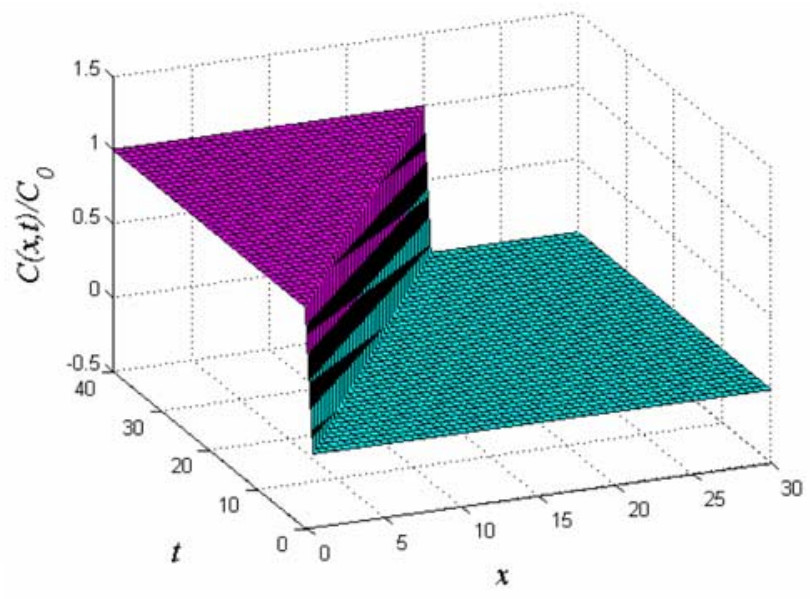

Explicit Taylor-Galerkin Scheme with $\theta=0$

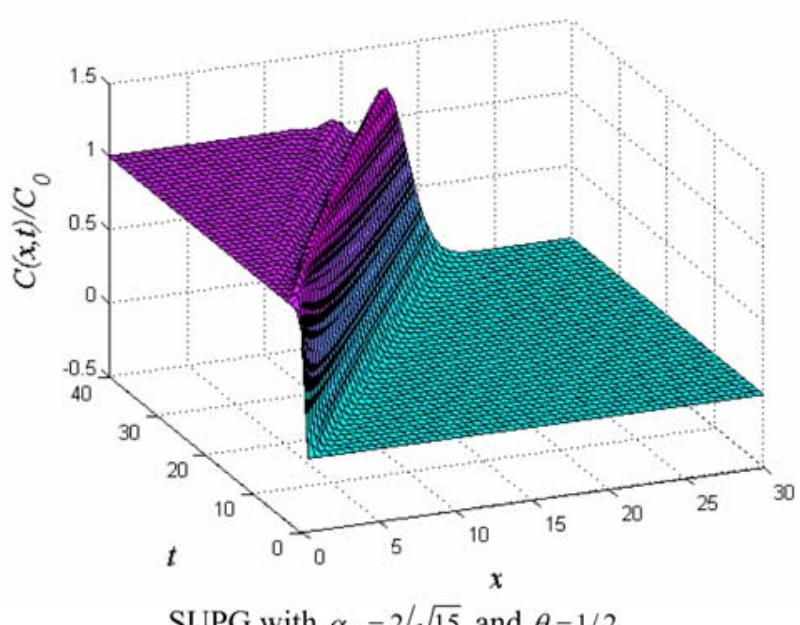

SUPG with $\alpha_{P}=2 / \sqrt{15}$ and $\theta=1 / 2$

Figure 1. Calibration of the computational schemes for the classical plug-flow problem.

The exact closed form solution for the initial boundary value problem defined by (15), (16) and (7) takes the form

$$
C(x, t)=C_{0} \exp (-\xi t) \mathrm{H}\left(\frac{\{1-\exp (-\lambda t)\}}{\lambda}-\frac{x}{v_{0}}\right) .
$$

\subsection{Plug Flow Problem for a Velocity Field With Exponential Time Decay, Natural Attenuation Within the Domain, and an Idealized Time-Dependent Boundary Concentration}

[13] The partial differential equation and the initial condition governing the chemical transport problem are given by (15) and (7) respectively and the time variation in the boundary concentration takes the form

$$
\begin{aligned}
C(0, t)= & C_{0}\left\{\exp \left(-\xi\left(\frac{\exp (-\lambda t)+\lambda t-1}{\lambda}\right)\right)\right. \\
& \left.\cdot \mathrm{H}\left(\frac{\{1-\exp (-\lambda t)\}}{\lambda}\right)\right\} .
\end{aligned}
$$

The exact closed form solution for the resulting initial boundary value problem is given by

$$
\begin{aligned}
C(x, t)= & C_{0}\left\{\exp \left(-\frac{\xi x}{v_{0}}-\xi\left(\frac{\exp (-\lambda t)+\lambda t-1}{\lambda}\right)\right)\right. \\
& \left.\cdot \mathrm{H}\left(\frac{[1-\exp (-\lambda t)]}{\lambda}-\frac{x}{v_{0}}\right)\right\} .
\end{aligned}
$$

\section{Computational Schemes}

[14] The exact closed form solutions presented here for a range of one-dimensional advective transport problems provide valuable benchmarking examples for testing the accuracy of computational schemes used to solve advective transport problems. There are a number of computational schemes available in the literature and a detailed discussion of these is perhaps not warranted within the context of this paper. We shall present here the salient features of three techniques used extensively to model advective transport problems. For brevity, we shall consider the homogeneous 


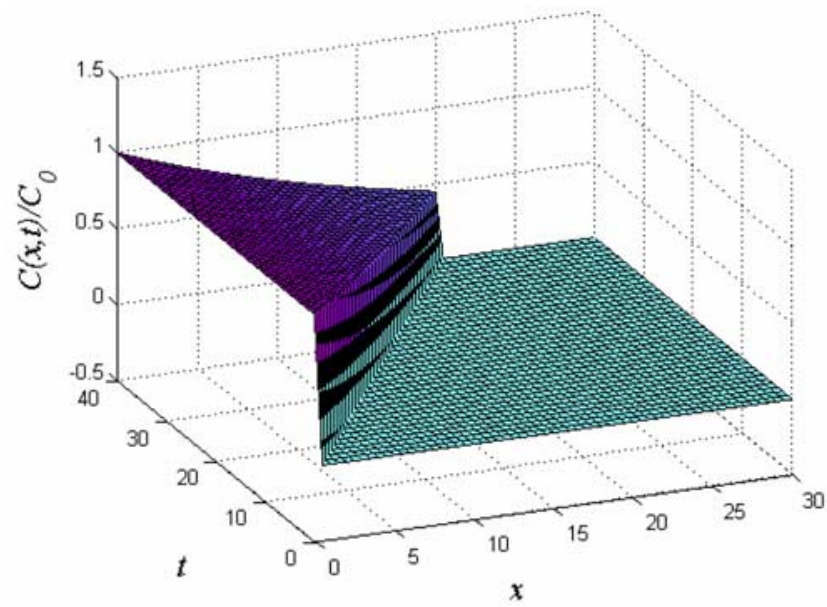

Analytical solution [Eq. (10)]

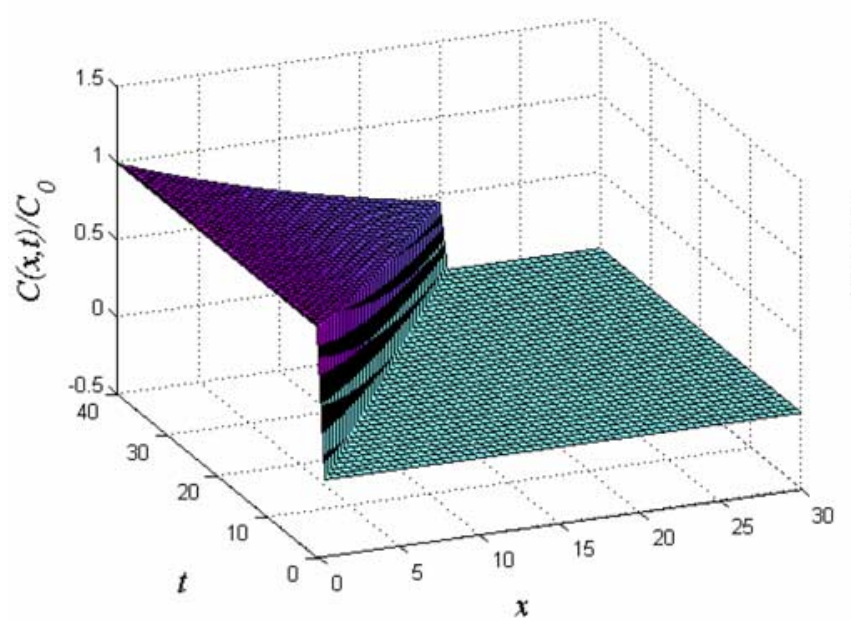

MLS with $\alpha=3 / 2$ and $\theta=1 / 3$

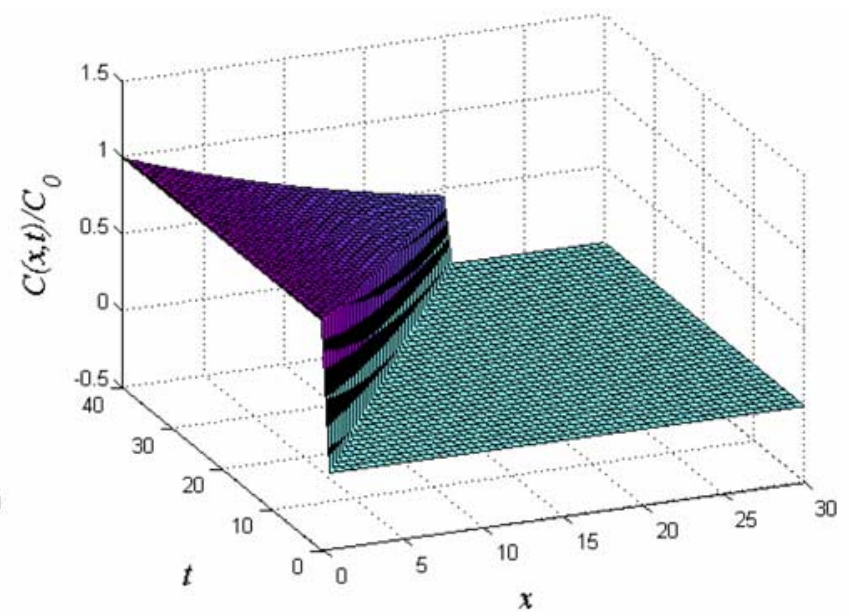

Explicit Taylor-Galerkin Scheme with $\theta=0$

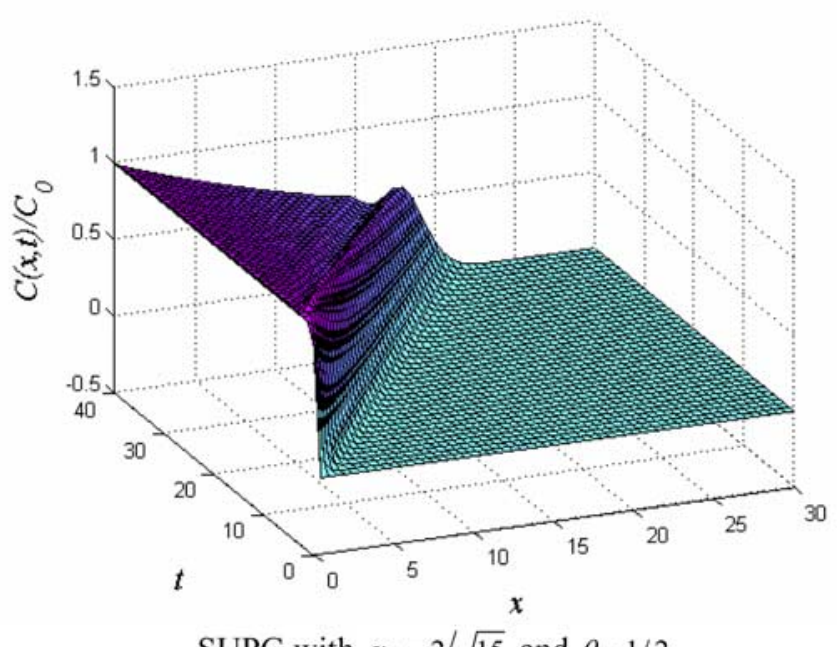

SUPG with $\alpha_{P}=2 / \sqrt{15}$ and $\theta=1 / 2$

Figure 2. Calibration of the computational schemes for the plug-flow problem with natural attenuation.

form of the advective transport equation and consider the application of the Euler time integration scheme along with the trapezoidal rule: i.e.,

$$
\frac{C^{n+1}-C^{n}}{\Delta t}+\mathbf{v} \nabla C^{n+\theta}=0
$$

where $C^{n+\theta}=(1-\theta) C^{n}+\theta C^{n+1}$, and $\theta \in(0,1)$ is the time weighting.

\subsection{Streamline Upwind Petrov-Galerkin Method}

[15] A smart upwind scheme can be introduced to the conventional finite element method by considering an asymmetric weighting function. The initial upwind finite element technique presented by Christie et al. [1976] for the one-dimensional advection-diffusion equation used a modified weighting function to achieve the upwind effect (i.e., the upstream element of a node was weighted more than the downstream element). The asymmetric weighting functions can be constructed by adding a perturbation to the standard Galerkin weighting function. Since such a perturbation needs to be incorporated only in the vicinity of a discontinuity in the solution or in a region with a high spatial gradient of the dependent variable, it should be related to the gradient of the weighting function $w$. Also, in order to avoid cross-wind diffusion in multidimensional problems, this perturbation should also be added in the flow direction. Hughes and Brooks [1982] developed the Streamline Upwind Petrov Galerkin (SUPG) technique for the computational modeling of the advection equation; the weightedresidual integral form can be written as

$$
\int_{V}\left(w+\frac{\alpha_{P} h}{2\|\mathbf{v}\|} \mathbf{v} \cdot \nabla w\right)\left(\frac{\partial C}{\partial t}+\mathbf{v} \cdot \nabla C^{n+\theta}\right) d V=0
$$

where $\left(\alpha_{P} h / 2\|\mathbf{v}\|\right) \mathbf{v} \cdot \nabla w$ is the perturbation added to the classical weighting function $w, h$ is a characteristic length of an element in the discretization scheme and $\alpha_{P}$ is a nondimensional upwind parameter. The results obtained by Christie et al. [1976] indicate that an optimum solution can be obtained by setting $\alpha_{P}=2 / \sqrt{15}$. This result has also been confirmed by Raymond and Garder [1976] on the basis of a Fourier analysis conducted on the spatial discretization of the governing equation. (For example, for the case of the steady state advection diffusion equation, the SUPG will be nodally exact on a piecewise element with the definition $\alpha_{P}=\operatorname{coth}(\mathrm{Pe})-(\mathrm{Pe})^{-1}$.) Other discontinuity 


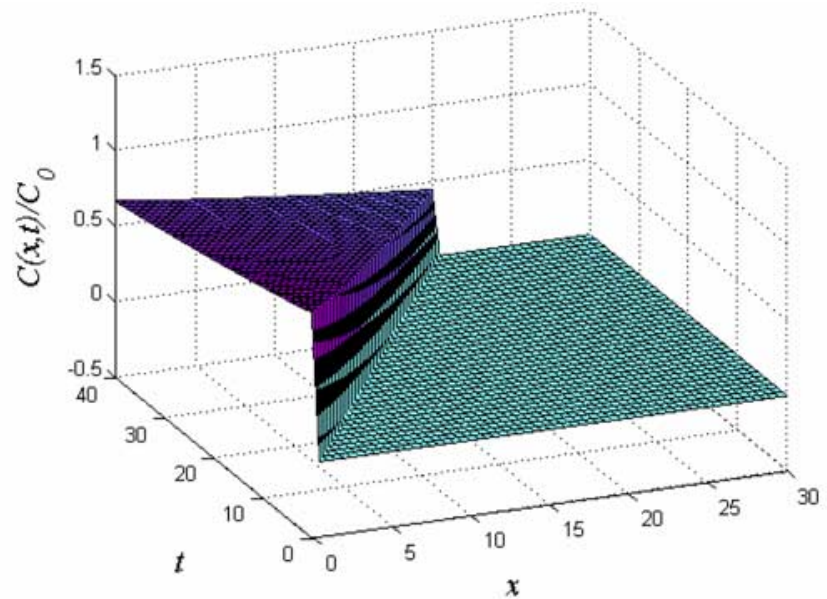

Analytical solution [Eq. (12)]

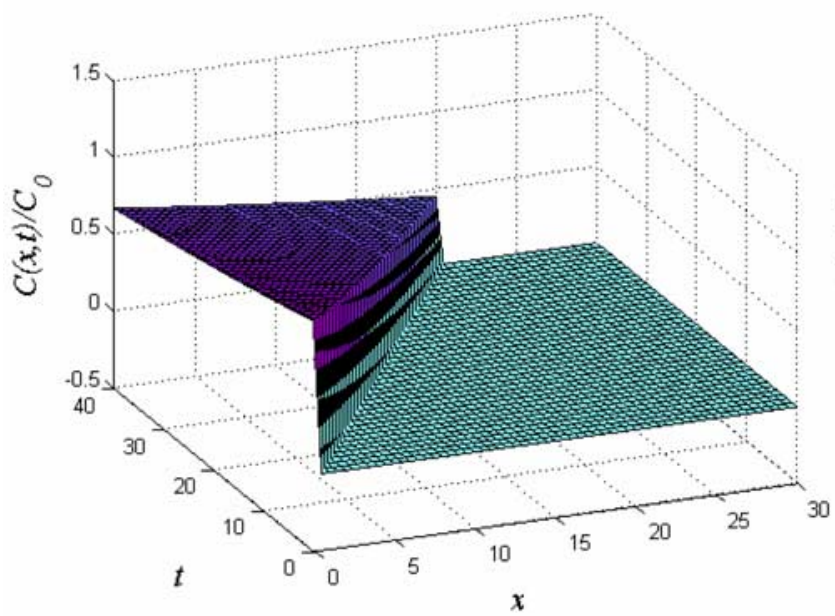

MLS with $\alpha=3 / 2$ and $\theta=1 / 3$

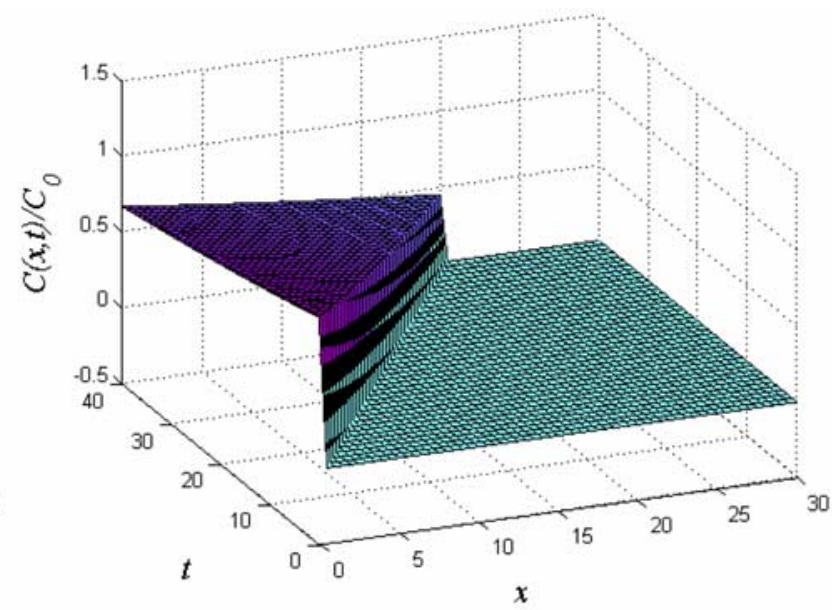

Explicit Taylor-Galerkin Scheme with $\theta=0$

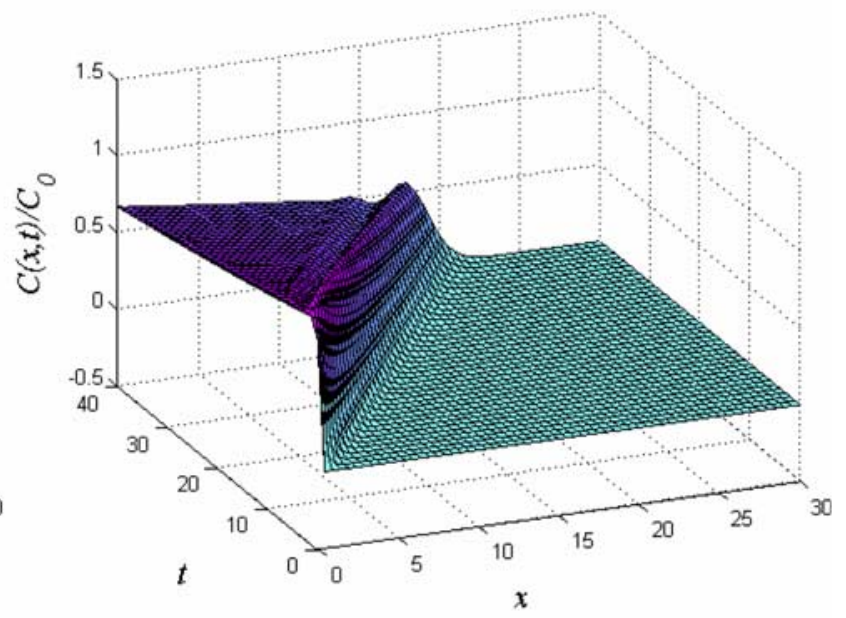

SUPG with $\alpha_{P}=2 / \sqrt{15}$ and $\theta=1 / 2$

Figure 3. Calibration of the computational schemes for the plug-flow problem with natural attenuation and exponential time decay in the boundary concentration.

capturing techniques have been incorporated into computational schemes to improve the performance of the SUPG scheme [Hughes and Mallet, 1986]. This involves the use of a term of the form $\alpha^{\prime} \mathbf{v}_{\mathrm{P}} \cdot \nabla w$, where $\alpha^{\prime}$ is a parameter and $\mathbf{v}_{\mathrm{P}}$ is defined as the projection of $\mathbf{v}$ on to the direction of $\nabla C$ with respect to the Riemann metric and is given by

$$
\begin{array}{ll}
\mathbf{v}_{\mathrm{P}}=\frac{(\mathbf{v} \cdot \nabla C)}{\|\nabla C\|^{2}} \nabla C & \text { if } \quad \nabla C \neq 0 \\
\mathbf{v}_{\mathrm{P}}=0 & \text { if } \quad \nabla C=0,
\end{array}
$$

which is a function of the gradient of the dependent variable, which in turn converts the original linear problem to a nonlinear one. In the computational results presented here only results derived from the regular SUPG scheme will be presented.

\subsection{Taylor-Galerkin Method}

[16] An alternative approach for developing a stabilized form for the advection equation is to consider a Taylor series expansion in time for the dependent variable to obtain a higher-order correction in time [Lax and Wendroff, 1960] and to use the Galerkin approach to generate a TaylorGalerkin weighted integral form [Donea, 1984; Donea et al., 1984]. For example, considering a Taylor series expansion of the dependent variable, at the $(n+1)$ th time step, we have

$$
C^{n+1}=C^{n}+\frac{\partial C^{n}}{\partial t} \Delta t+\frac{1}{2} \frac{\partial^{2} C^{n}}{\partial t^{2}}(\Delta t)^{2}+\frac{1}{6} \frac{\partial^{3} C^{n}}{\partial t^{3}}(\Delta t)^{3}+\mathrm{O}\left((\Delta t)^{4}\right) .
$$

When terms up to the second-order of the above expansion are considered, a stabilized equation of the following form is obtained:

$$
\frac{C^{n+1}-C^{n}}{\Delta t}+\mathbf{v} \cdot \nabla C^{n}=\frac{\Delta t}{2}\|\mathbf{v}\|^{2} \nabla^{2} C^{n}
$$

Applying a standard Galerkin technique to the above equation gives a weak form of the second-order Taylor- 


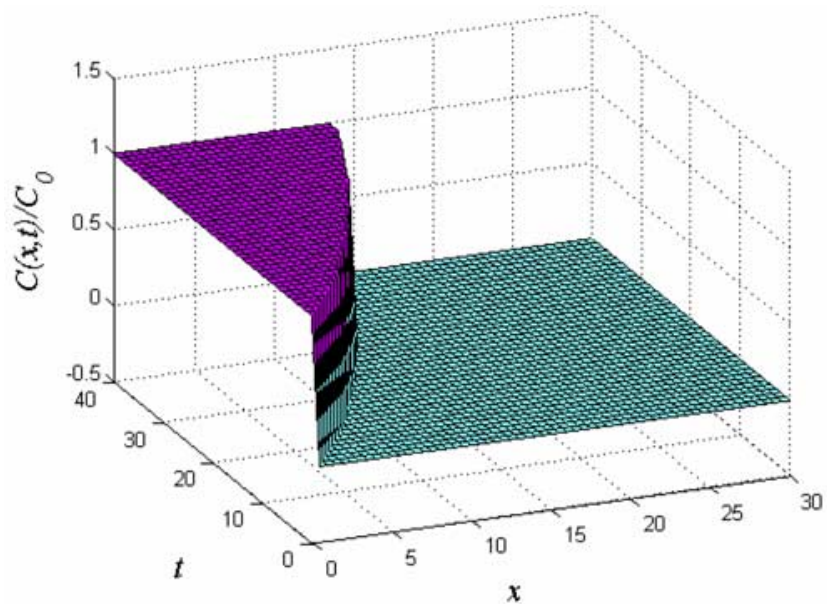

Analytical solution [Eq. (14)]

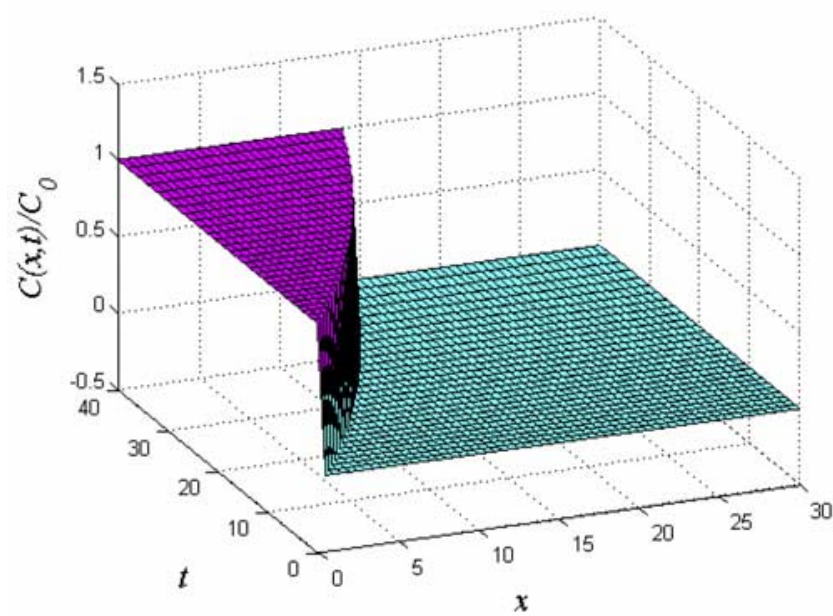

MLS with $\alpha=3 / 2$ and $\theta=1 / 3$

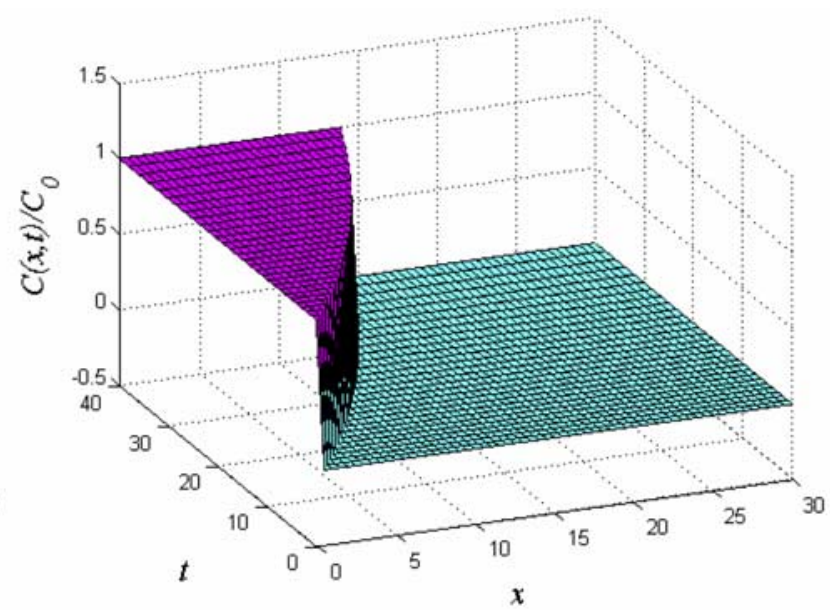

Explicit Taylor-Galerkin Scheme with $\theta=0$

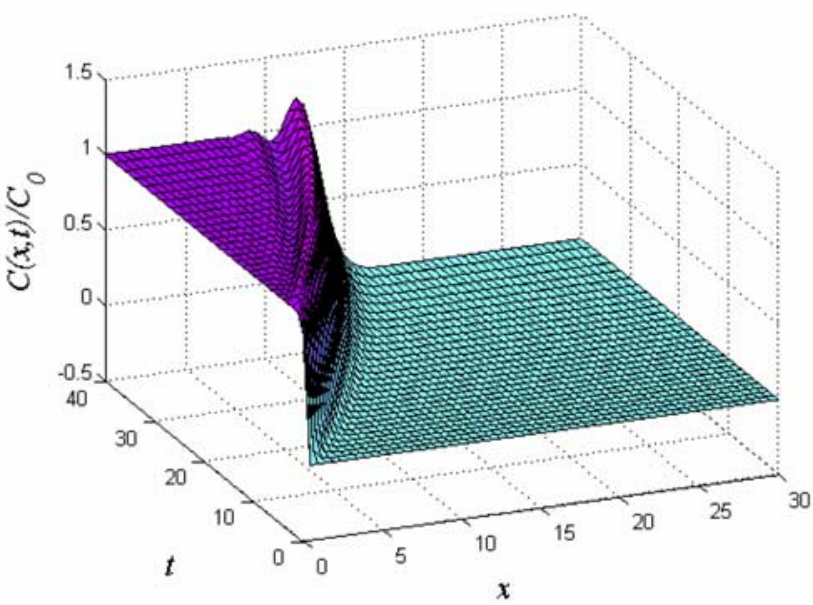

SUPG with $\alpha_{P}=2 / \sqrt{15}$ and $\theta=1 / 2$

Figure 4. Calibration of the computational schemes for the plug-flow problem with exponential time decay in the flow velocity.

Galerkin scheme for the solution of the advection equation, expressed as

$$
\int_{V}\left(\frac{C^{n+1}-C^{n}}{\Delta t}\right) d V+\int_{V}\left(w+\frac{\Delta t}{2} \mathbf{v} \cdot \nabla w\right)\left(\mathbf{v} \cdot \nabla C^{n+\theta}\right) d V=0 .
$$

Similarly, if terms of order up to the third in $\Delta t$ are considered, the resulting stabilized equation can be expressed as

$$
\left(1-\frac{(\Delta t)^{2}}{6}\|\mathbf{v}\|^{2} \nabla^{2}\right)\left(\frac{C^{n+1}-C^{n}}{\Delta t}\right)+\mathbf{v} \cdot \nabla C^{n}=\frac{(\Delta t)^{2}}{2}\|\mathbf{v}\|^{2} \nabla^{2} C^{n},
$$

and the corresponding weak form of the advection equation takes the form

$$
\begin{gathered}
\int_{V}\left(w+\frac{(\Delta t)^{2}}{6}\|\mathbf{v}\|^{2} \nabla w \cdot \nabla\right)\left(\frac{C^{n+1}-C^{n}}{\Delta t}\right) d V \\
+\int_{V}\left(w+\frac{\Delta t}{2} \mathbf{v} \cdot \nabla w\right)\left(\mathbf{v} \cdot \nabla C^{n+\theta}\right) d V=0 .
\end{gathered}
$$

It is evident from (27) that an artificial diffusion term is added to the original advection equation owing to the Taylor expansion scheme. From (27) it is evident that the expansion scheme also gives an artificial convection term in the residual integral form (second integral of (27) due to the incorporation of higher-order terms in (23).

\subsection{Least Squares Method}

[17] A third approach for deriving a weighted-residual integral of the form (21) proposed by Carey and Jiang [1987a, 1987b, 1988] and Jiang [1998] considers the least squares functional of the residual integral of the advection equation, which can be written as

$$
J\left(C^{n+1}\right)=\frac{1}{2} \int_{V}\left(\frac{C^{n+1}-C^{n}}{\Delta t}+\mathbf{v} \cdot \nabla C^{n+\theta}\right)^{2} d V .
$$

Using the stationary condition $\delta J=0$ with respect to the arguments $C^{n+1}$, and replacing the variation in the dependent variable $\delta C^{n+1}$ by the weighting function $w$, 


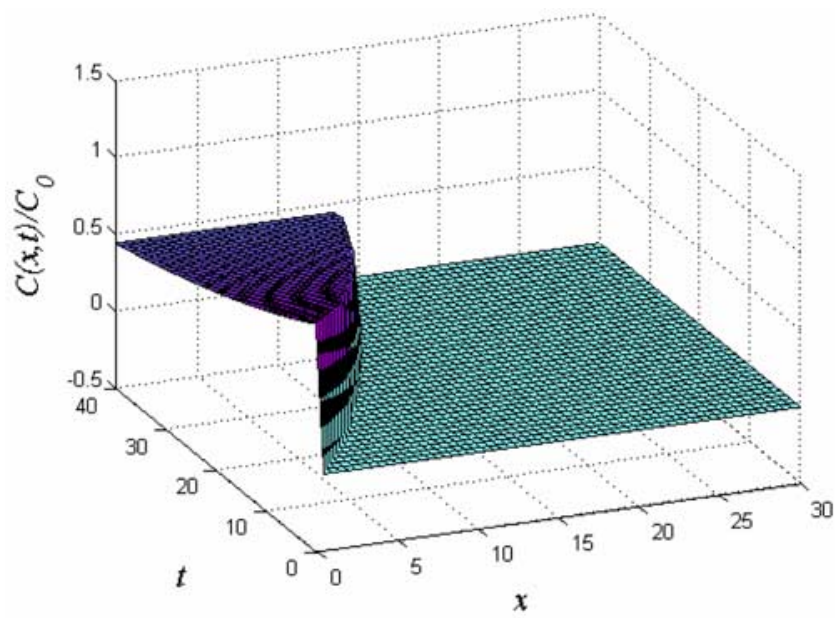

Analytical solution [Eq. (17)]

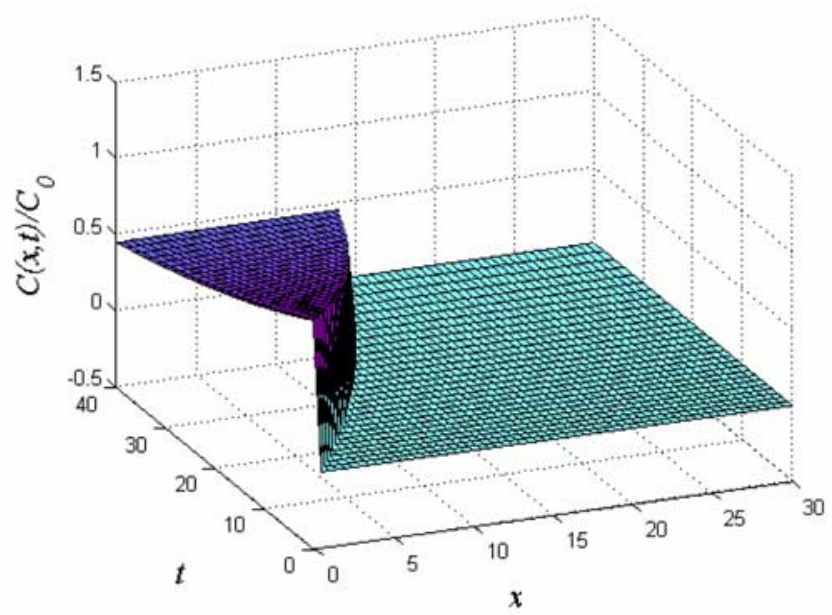

MLS with $\alpha=3 / 2$ and $\theta=1 / 3$

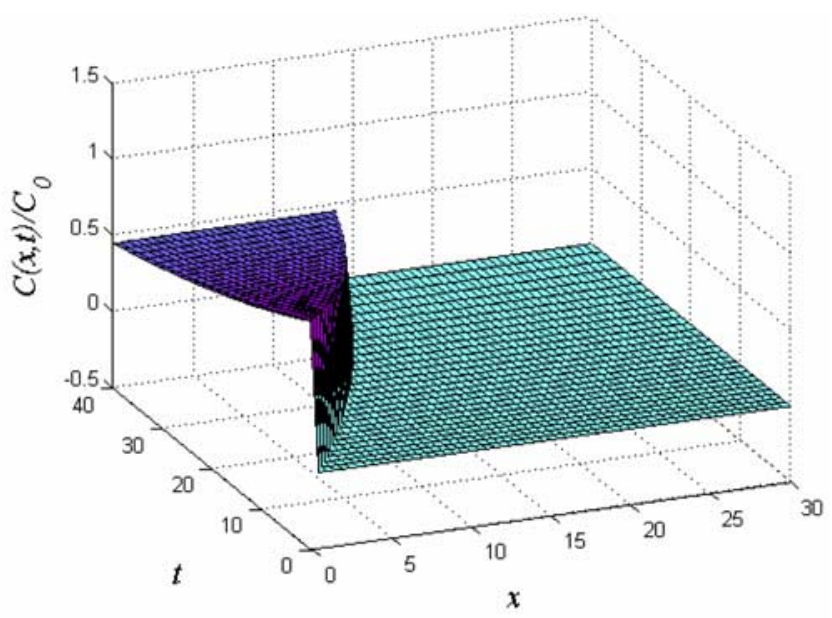

Explicit Taylor-Galerkin Scheme with $\theta=0$

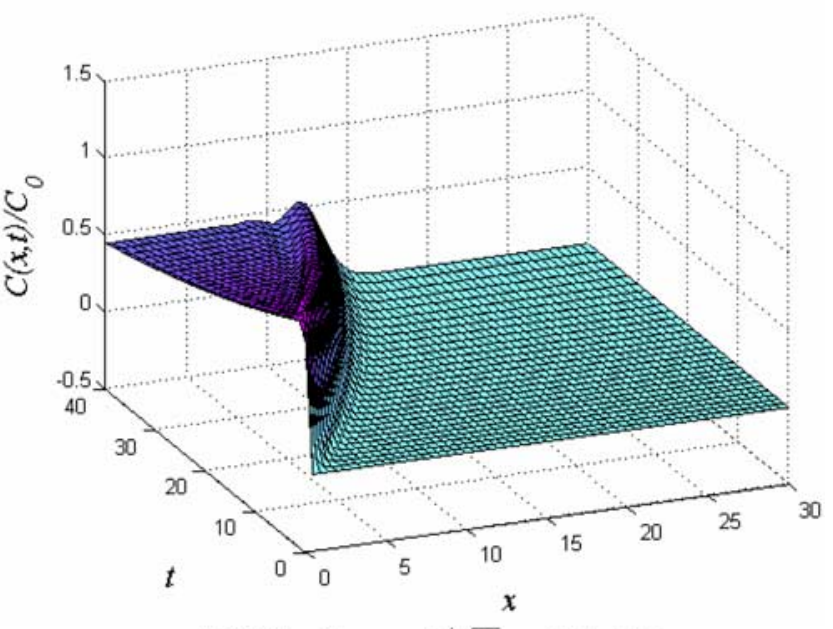

SUPG with $\alpha_{P}=2 / \sqrt{15}$ and $\theta=1 / 2$

Figure 5. Calibration of the computational schemes for the plug-flow problem with natural attenuation, natural boundary attenuation, and exponential time decay in the flow velocity.

we can construct a weighted-residual integral form of the advection equation, which can be written as

$$
\int_{V}[w+\theta(\Delta t) \mathbf{v} \cdot \nabla w]\left(\frac{C^{n+1}-C^{n}}{\Delta t}+\mathbf{v} \cdot \nabla C^{n+\theta}\right) d V=0 .
$$

In comparison to the classical Galerkin technique, a perturbation with the form of $\theta(\Delta t) \mathbf{v} \cdot \nabla w$ is added to the weighting function and the corresponding scheme can give rise to a symmetric form of a matrix equation, which has advantages in the solution of problems involving nonlinear transport phenomena.

[18] The Symmetric Streamline Stabilization technique proposed by Wendland and Schmid [2000] for strongly advection dominated processes, introduces an upwind term into the least squares technique to obtain a better computational performance in advection-dominated processes. This is equivalent to using different perturbation parameters in the least squares scheme for the temporal and spatial terms of the advection equation. The resulting weighted integral form can be written as

$$
\begin{aligned}
\int_{V}[w+\theta(\Delta t) \mathbf{v} \cdot \nabla w]\left(\frac{C^{n+1}-C^{n}}{\Delta t}\right) d V \\
\quad+\int_{V}[w+\alpha \theta(\Delta t) \mathbf{v} \cdot \nabla w] \mathbf{v} \cdot \nabla C^{n+\theta} d V=0 .
\end{aligned}
$$

In (30), $\alpha$ is the upwind parameter that can be determined using a Fourier analysis, which ensures optimal numerical performance of the Modified Least Squares scheme for the advection equation. Further discussion of these aspects are given by Selvadurai and Dong [2006a, 2006b].

\section{A Comparison of Analytical Results and Computational Estimates}

[19] The computational performance of the procedures described in section 4 was examined using the analytical solutions presented in section 3. The computational domain 


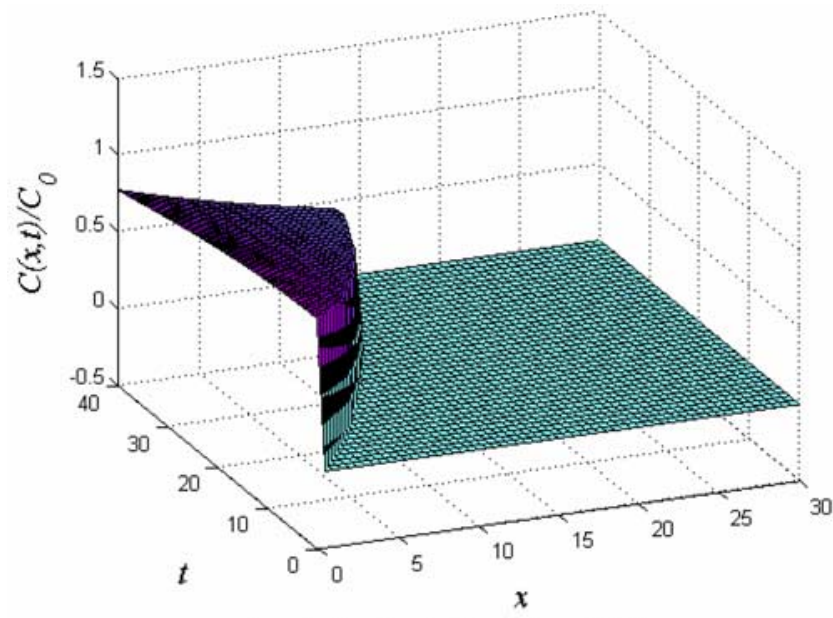

Analytical solution [Eq. (19)]

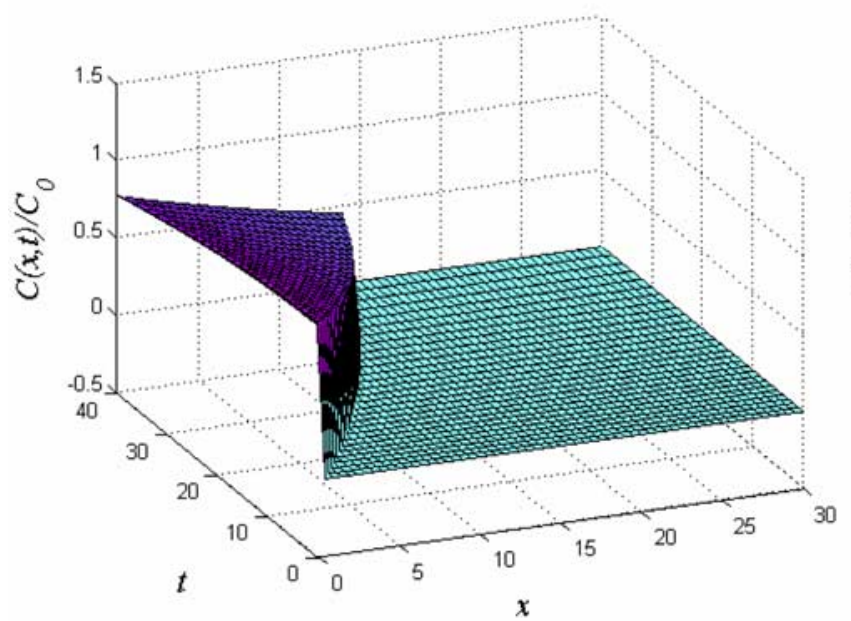

MLS with $\alpha=3 / 2$ and $\theta=1 / 3$

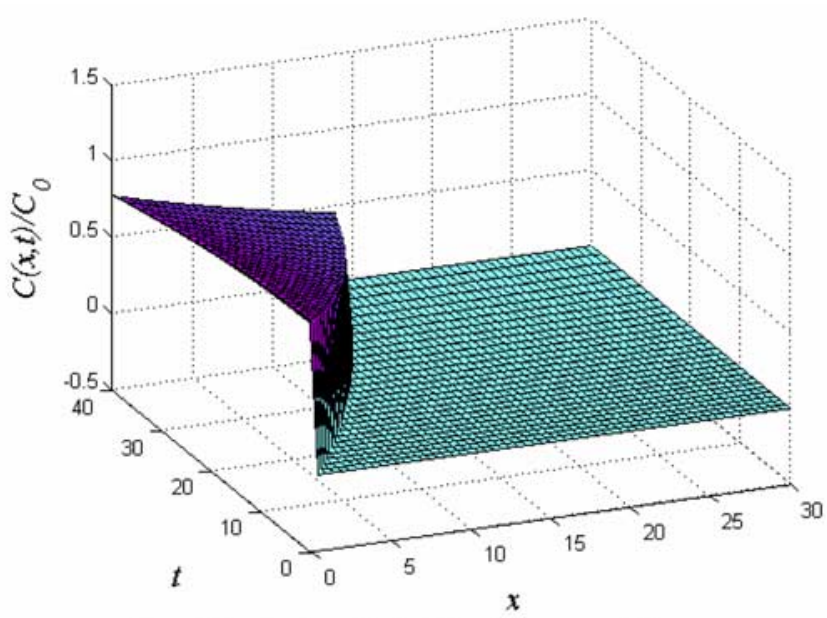

Explicit Taylor-Galerkin Scheme with $\theta=0$

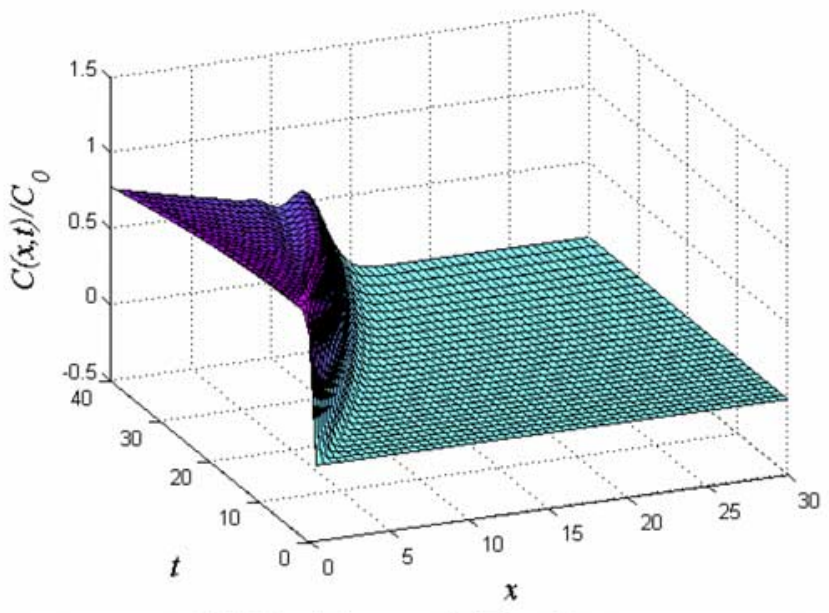

SUPG with $\alpha_{P}=2 / \sqrt{15}$ and $\theta=1 / 2$

Figure 6. Calibration of the computational schemes for the plug-flow problem with natural attenuation, exponential boundary attenuation of concentration and exponential time decay in the flow velocity.

is set at a finite value of $[0,30 \mathrm{~m}]$ and the flow velocity is set at $v_{0}=0.5 \mathrm{~m} / \mathrm{sec}$. The domain is discretized into 60 elements of length $\mathrm{h}=0.5 \mathrm{~m}$. The time step is taken as $\Delta t=$ $1.0 \mathrm{sec}$, such that the Courant number $\mathrm{Cr}=\left(v_{0} \Delta t / h\right)=1$. Figures 1 and 2 illustrate the comparisons for the plug flow problem with constant boundary potential either in the absence or in the presence of natural attenuation. Also in Figure 2, the natural attenuation factor is taken as $\xi=0.02$. The computational results derived from the Taylor-Galerkin scheme and Modified Least Squares scheme show an accuracy of approximately $0.5 \%$ compared with the exact closed form analytical result. A Fourier analysis [Selvadurai and Dong, 2006b] of the Modified Least Squares scheme indicates that an optimum performance of the scheme indicated by (29) is obtained when $\alpha=3 / 2$ and $\theta=1 / 3$. These figures also present results derived from the PetrovGalerkin technique where the value of $\alpha_{P}$ in (21) is set to $2 / \sqrt{15}$ and $\theta$ is set to $1 / 2$. The results derived from the Streamline Upwind Petrov-Galerkin (SUPG) scheme exhibit oscillatory behavior in regions to the rear of the discontinuous front and numerical dissipation ahead of the discontinuous front. In the case of the classical plug flow problem (Figure 1), the observed oscillations give rise to discrepancies of approximately $21.66 \%$ between the SUPG solution and the classical analytical result, at the termination of the computations at $40 \mathrm{~s}$. When natural attenuation is present (Figure 2) the discrepancy between the SUPG result and the analytical result becomes as large as 33.33\% at the $40 \mathrm{~s}$ mark. The computational results also display a progressively diffusive pattern at the discontinuous front although there is no simple measure that can be used to quantify the diffusive pattern. Figure 3 illustrates comparisons for the advective transport problems where there is natural attenuation and a decay in the boundary concentration specified at $\beta=0.01$. The comparisons indicate that the results derived from the Taylor-Galerkin and Modified Least Squares techniques compare to within an accuracy of approximately $0.5 \%$ with the analytical solution. The results derived from the SUPG scheme displays oscillatory and dissipative phenomena, respectively, to the rear and ahead of the discontinuous front, with the peaks differing from the analytical solution by as much as $21 \%$ at the $40 \mathrm{~s}$ mark. Figure 4 illustrates the results for the plug flow problem where the advective flow velocity exhibits expo- 
nential time decay as defined by (14). The decay parameter is taken as $\lambda=0.02$. Here again, the Taylor-Galerkin and Modified Least Squares techniques provide the best correlations with the discrepancy within $0.5 \%$. The SUPG technique gives strong oscillations at the trailing part of the discontinuity with an overestimation of $21.66 \%$ at the termination of computations at $40 \mathrm{~s}$. Figure 5 considers the case where plug flow takes place in the presence of natural attenuation, specified boundary attenuation and an exponential decay in the advective flow velocity. The parameters chosen for the decay terms are $\xi=0.02$ and $\lambda=$ 0.02 . The comparisons between the analytical solution and the computational estimates based on the Taylor-Galerkin and the Modified Least Squares techniques indicate that the computational schemes can match the analytical solution to within $0.5 \%$, whereas the results derived from the SUPG scheme exhibits the characteristic oscillatory phenomena at the trailing edge of the discontinuous front, which leads to discrepancies of approximately $26.92 \%$ at $40 \mathrm{~s}$ and dissipation ahead of the front. Figure 6 illustrates comparisons for the plug flow problem where there is natural attenuation, exponential boundary attenuation of concentration and exponential time decay in the flow velocity. The parameters chosen for the decay terms are $\xi=0.02$ and $\lambda=0.02$. Here again, the results derived from the Taylor-Galerkin and Modified Least Squares techniques offer the best correlations with the exact analytical result, giving a maximum discrepancy between the analytical and computational results of less than $0.5 \%$. The results derived from the SUPG scheme exhibit the usual oscillations and diffusion, respectively, to the rear and ahead of the discontinuity with the maximum discrepancy being approximately $29.63 \%$ at $40 \mathrm{~s}$.

\section{Concluding Remarks}

[20] The development of reliable computational schemes for the modeling of advection-dominated problems is important to the application of such methods to practical situations involving the water borne migration of contaminants, chemicals and particulates in fluid-saturated porous media. The reliability of most computational schemes is usually assessed through their ability to model purely advective transport problems that often give rise to concentration profiles involving a discontinuous contaminant migration front. The conventional discontinuous advective transport involves the one-dimensional plug flow problem. This paper presents new one-dimensional solutions for the plug flow problem that incorporate natural attenuation, timedependent boundary concentrations and time-dependent advective flow velocities. These analytical solutions are used to assess the computational predictions of three computational schemes involving the Taylor-Galerkin, the Streamline Upwind Petrov-Galerkin and the Modified Least Squares methods. The comparisons indicate that the TaylorGalerkin and Modified Least Squares techniques provide better computational simulations of the plug flow problem with a discrepancy of less than $0.5 \%$ between the analytical and computational estimates. In contrast, the conventional Streamline Upwind Petrov-Galerkin scheme gives rise to both dispersive and oscillatory concentrations in the neighborhood of the discontinuous front. The discrepancies can range from peaks that are between $21.66 \%$ and $33.33 \%$ of the exact solution, for a simulation that lasts $40 \mathrm{~s}$, depending on the particular initial boundary value problem that is being examined. Furthermore the discrepancies will be amplified when the simulations are performed for a longer duration. The analytical results presented provide a departure from the traditional plug flow problem that is void of features related to other time-dependent effects, including time variable velocities, time variable boundary inputs and natural attenuation, which can have a significant influence the performance of the computational approach.

[21] Acknowledgments. The author is indebted to the reviewers for their detailed comments that lead to improvements in the presentation. The work described in this paper was supported by the Max Planck Forschungspreis in the Engineering Sciences awarded to the author by the Max Planck Gesellschaft, Germany. The author is grateful to W. Dong for his assistance with the computations.

\section{References}

Appelo, C. A. J., and D. Postma (1993), Geochemistry, Groundwater and Pollution, A.A. Balkema, Rotterdam, Netherlands.

Banks, R. B. (1994), Growth and Diffusion Phenomena: Mathematical Frameworks and Applications, Springer, Berlin.

Bear, J. (1972), Dynamics of Fluids in Porous Media, Dover, Mineola, N. Y. Bear, J., and Y. Bachmat (1992), Introduction to the Modelling of Transport Phenomena in Porous Media, D. Reidel, Dordrecht, Netherlands.

Bear, J., and A. Verruijt (1990), Modelling of Groundwater Flow and Pollution, D. Reidel, Dordrecht, Netherlands.

Bedient, P. B., H. S. Rifai, and C. J. Newell (1999), Groundwater Contamination: Transport and Remediation, Prentice-Hall, Upper Saddle River, N. J.

Boztosun, I., and A. Charafi (2002), An analysis of the linear advectiondiffusion equation using mesh-free and mesh-dependent methods, Eng. Anal. Boundary Elements, 26, 889-895, doi:10.1016/S0955-7997(02) 00053-X.

Carey, G. F., and B. N. Jiang (1987a), Least squares finite element method and pre-conditioned conjugate gradient solution, Int. J. Numer. Methods Eng., 24, 1283-1296, doi:10.1002/nme.1620240705.

Carey, G. F., and B. N. Jiang (1987b), Nonlinear pre-conditioned conjugate gradient and the least squares finite elements, Comput. Methods Appl. Mech. Eng., 62, 145-154, doi:10.1016/0045-7825(87)90020-X.

Carey, G. F., and B. N. Jiang (1988), Least square finite elements for firstorder hyperbolic systems, Int. J. Numer. Methods Eng., 26, 81-93, doi:10.1002/nme.1620260106.

Charbeneau, R. J. (2000), Groundwater Hydraulics and Pollutant Transport, Prentice-Hall, Upper Saddle River, N. J.

Christie, I., D. Griffiths, A. Mitchell, and O. C. Zienkiewicz (1976), Finite element methods for second-order differential equations with significant first derivatives, Int. J. Numer. Methods Eng., 10, 1389-1396, doi:10.1002/ nme.1620100617.

de Marsily, G. (1986), Quantitative Hydrogeology: Groundwater Hydrology for Engineers, Academic, San Diego, Calif.

Domenico, P. A., and F. W. Schwartz (1998), Physical and Chemical Hydrogeology, John Wiley, Hoboken, N. J.

Donea, J. A. (1984), Taylor-Galerkin method for convective transport problems, Int. J. Numer. Methods Eng., 20, 101-119, doi:10.1002/nme. 1620200108

Donea, J., S. Giulian, H. Laval, and L. Quartapelle (1984), Time-accurate solution of advection-diffusion problem by finite elements, Comput. Methods Appl. Mech. Eng., 45, 123-145, doi:10.1016/0045-7825(84)90153-1.

Douglas, J., Jr., and T. F. Russell (1982), Numerical methods for convection-dominated diffusion problems based on combining the methods of characteristics with finite element or finite difference procedures, SIAM J. Numer. Anal., 19, 871-885, doi:10.1137/0719063.

Fetter, C. W. (1993), Contaminant Hydrogeology, Macmillan, New York. Greenkorn, R. A. (1983), Flow Phenomena in Porous Media, Marcel Dekker, New York.

Hauke, G., and M. H. Doweidar (2005), Fourier analysis of semi-discrete and space-stabilized methods for the advective-diffusive-reactive equation: II SGS, Comput. Methods Appl. Mech. Eng., 194, 691-725, doi:10.1016/j.cma.2004.06.005.

Hughes, T. J. R., and A. Brooks (1982), A theoretical framework for Petrov-Galerkin methods with discontinuous weighting functions: Application to the Streamline-Upwind Procedure, in Finite Elements in Fluids, 
vol. 4, edited by R. H. Gallagher et al., pp. 47-65, John Wiley, Chichester, U.K.

Hughes, T. J. R., and M. Mallet (1986), A new finite element formulation for computational fluid dynamics. IV. A discontinuity-capturing operator for multi-dimensional advective-diffusive systems, Comput. Methods Appl. Mech. Eng., 58, 329-336, doi:10.1016/0045-7825(86)90153-2.

Huyakorn, P. S., and G. F. Pinder (1983), Computational Methods in Subsurface Flows, Academic, San Diego, Calif.

Ingebritsen, S. E., and W. D. Sanford (1998), Groundwater in Geologic Processes, Cambridge Univ. Press, Cambridge, U.K.

Ingham, D. B., and I. Pop (Eds.) (1998), Transport Phenomena in Porous Media, Elsevier, New York.

Jiang, B. N. (1998), The Least-Squares Finite Element Method: Theory and Application in Computational Fluid Dynamics and Electromagnetics, Springer, Berlin.

Lax, P. D., and B. Wendroff (1960), Systems of conservation laws, Commun. Pure Appl. Math., 13, 217-237, doi:10.1002/cpa.3160130205.

LeVeque, R. (2002), Finite Volume Methods for Hyperbolic Problems, Cambridge Univ. Press, Cambridge, U.K.

Lindstrom, F. T., and F. Oberhettinger (1975), A note on a Laplace transform pair associated with mass transport in porous media and heat transport problems, SIAM J. Appl. Math., 29, 288-292, doi:10.1137/ 0129026.

Lindstrom, F. T., and W. M. Stone (1974a), On the start up or initial phase of linear mass transport of chemicals in a water saturated sorbing porous medium. I, SIAM J. Appl. Math., 26, 578-591, doi:10.1137/0126055.

Lindstrom, F. T., and W. M. Stone (1974b), On the start up or initial phase of linear mass transport of chemicals in a water saturated sorbing porous medium. II: Integral equation approach, SIAM J. Appl. Math., 26, $592-$ 606, doi:10.1137/0126056.

Lindstrom, F. T., R. Haque, V. H. Freed, and L. Boersma (1967), Theory on the movement of some herbicides in soils: Linear diffusion and convection of chemicals in soils, Environ. Sci. Technol., 1, 561-565, doi:10.1021/es60007a001.

Massel, S. R. (1999), Fluid Mechanics for Marine Ecologists, Springer, Berlin.

McDowell-Boyer, L. M., J. R. Hunt, and N. Sitar (1986), Particle transport through porous media, Water Resour. Res., 22, 1901-1921, doi:10.1029/ WR022i013p01901.

Morton, K. W. (1998), On the analysis of finite volume methods for evolutionary problems, SIAM J. Numer. Anal., 35, 2195-2222, doi:10.1137/ S0036142997316967.

Neuman, S. P. (1981), An Eulerian-Lagrangian numerical scheme for the dispersion convection equation using conjugate space time grids, J. Comput. Phys., 41, 270-294, doi:10.1016/0021-9991(81)90097-8.

Neuman, S. P. (1984), Adaptive Eulerian-Lagrangian finite element method for advection-dispersion, Int. J. Numer. Methods Eng., 20, 321-337, doi:10.1002/nme.1620200211.

Noorishad, J., C. F. Tsang, P. Perrochet, and A. Musy (1992), A perspective on the numerical solution of convection-dominated problems: A price to pay for the easy way out, Water Resour. Res., 28, 551-562, doi:10.1029/ 91WR02730.

Oelkers, E. H. (1996), Physical and chemical properties of rocks and fluids for chemical mass transport calculations, in Reactive Transport in Porous Media, edited by P. C. Lichtner, C. I. Steefel, and E. H. Oelkers, Rev. Mineral., 34, 131-191.

Ogata, A., and R. B. Banks (1961), A solution of the differential equation of longitudinal dispersion in porous media, Geol. Surv. Prof. Pap., 411-a, A1-A7.

Oňate, E. (1998), Derivation of stabilized equations for numerical solution of advective-diffusive transport and fluid flow problems, Comput. Methods Appl. Mech. Eng., 151, 233-265, doi:10.1016/S0045-7825(97) 00119-9.

Pang, L., and B. Hunt (2001), Solutions and verifications of a scale-dependent dispersion model, J. Contam. Hydrol., 53, 21-39, doi:10.1016/ S0169-7722(01)00134-6.

Pereira, J. M. C., and J. C. F. Pereira (2001), Fourier analysis of several finite difference schemes for the one-dimensional unsteady convectiondiffusion equation, Int. J. Numer. Methods Fluids, 36, 417-439, doi:10.1002/fld.136.

Philips, O. M. (1991), Flow and Reactions in Permeable Rocks, Cambridge Univ. Press, Cambridge, U.K.

Raymond, W. H., and A. Garder (1976), Selective damping in a Galerkin method for solving wave problems with variable grids, Mon. Weather Rev.,
104,1583 - 1590, doi:10.1175/1520-0493(1976)104<1583:SDIAGM> 2.0.CO;2.

Rubin, J. (1983), Transport of reacting solutes in porous media: Relation between mathematical nature of problem formulation and chemical nature of reactions, Water Resour. Res., 19, 1231-1252, doi:10.1029/ WR019i005p01231.

Selvadurai, A. P. S. (2000), Partial Differential Equations in Mechanics, vol. 1, Fundamentals, Laplace's Equation, Diffusion Equation, Wave Equation, Springer, Berlin.

Selvadurai, A. P. S. (2002), Advective transport of a chemical from a cavity in a porous medium, Comput. Geotech., 29, 525-546, doi:10.1016/ S0266-352X(02)00007-1.

Selvadurai, A. P. S. (2003), Contaminant migration from an axisymmetric source in a porous medium, Water Resour. Res., 39(8), 1203, doi:10.1029/ 2002WR001442.

Selvadurai, A. P. S. (2004a), Advective transport from a penny-shaped crack and an associated uniqueness theorem, Int. J. Numer. Anal. Methods Geomech., 28, 191-208, doi:10.1002/nag.331.

Selvadurai, A. P. S. (2004b), On the advective-diffusive transport in porous media in the presence of time-dependent velocities, Geophys. Res. Lett., 31, L13505, doi:10.1029/2004GL019646.

Selvadurai, A. P. S. (2004c), On the uniqueness theorem for advectivediffusive transport in porous media: A canonical proof, Transp. Porous Media, 56, 51-60, doi:10.1023/B:TIPM.0000018404.42758.fc.

Selvadurai, A. P. S. (2006), Gravity-driven advective transport during deep geological disposal of contaminants, Geophys. Res. Lett., 33, L08408, doi:10.1029/2006GL025944.

Selvadurai, A. P. S., and W. Dong (2006a), A time-adaptive scheme for the accurate solution of the advection equation with a transient flow velocity, Comput. Model. Eng. Sci., 12, 41-53.

Selvadurai, A. P. S., and W. Dong (2006b), Numerical modeling of advective transport in the presence of fluid pressure gradients, Int. J. Numer. Anal. Methods Geomech., 30, 615-634, doi.10.1002/nag 494.

Singh, K. M., and M. Tanaka (2000), On exponential variable transformation based boundary element formulation for advection-diffusion problems, Eng. Anal. Boundary Elements, 24, 225-235, doi:10.1016/ S0955-7997(00)00003-5.

Sun, N.-Z. (1996), Mathematical Modeling of Groundwater Pollution, Springer, Berlin.

Taigbenu, A. E. (1999), Three Green element models for the diffusionadvection equation and their stability characteristics, Eng. Anal. Boundary Elements, 23, 577-589, doi:10.1016/S0955-7997(99)00005-3.

van Genuchten, M. T. (1981), Analytical solutions for chemical transport with simultaneous adsorption, zero-order production and first-order decay, J. Hydrol., 49, 213-233, doi:10.1016/0022-1694(81)90214-6.

Vukovich, M. (1997), Groundwater Dynamics: Steady Flow, Water Resour. Publ., Littleton, Colo.

Vulliet, L., L. Laloui, and B. A. Schrefler (Eds.) (2002), Environmental Geomechanics, Ecole Polytech. Fed. de Lausanne Press, Lausanne, Switzerland.

Wang, Y., and K. Hutter (2001), Comparisons of numerical methods with respect to convectively dominated problems, Int. J. Numer. Methods Fluids, 37, 721-745, doi:10.1002/fld.197.

Weinberger, H. F. (1965), A First Course in Partial Differential Equations, Dover, Mineola, N. Y.

Wendland, E., and G. A. Schmid (2000), Symmetrical streamline stabilization scheme for high advective transport, Int. J. Numer. Anal. Methods Geomech., 24, 29-45, doi:10.1002/(SICI)1096-9853(200001)24:1 $<29:: A I D-N A G 51>3.0 . C O ; 2-F$.

Young, D. L., Y. F. Wang, and T. I. Eldho (2000), Solution of the advectiondiffusion equation using the Eulerian-Lagrangian boundary element method, Eng. Anal. Boundary Elements, 24, 449-457, doi:10.1016/ S0955-7997(00)00026-6.

Yu, C. C., and J. C. Heinrich (1986), Petrov-Galerkin methods for the timedependent convective transport equation, Int. J. Numer. Methods Eng., 23, 883-901, doi:10.1002/nme.1620230510.

Zheng, C., and G. D. Bennett (2002), Applied Transport Modelling, Wiley Intersci., Hoboken, N. J.

A. P. S. Selvadurai, Department of Civil Engineering and Applied Mechanics, McGill University, Sherbrooke Street, West Montreal, QC H3A 2K6, Canada. (patrick.selvadurai@mcgill.ca) 